\title{
Recruiting a transcription factor in the liver to prevent atherosclerosis
}

\author{
Alan D. Attie \\ Department of Biochemistry, University of Wisconsin-Madison, Madison, Wisconsin, USA.
}

\begin{abstract}
Hypertriglyceridemia is associated with obesity, diabetes, and atherosclerosis. While lipoprotein lipase (LPL) hydrolyzes triglyceride (TC) cargo into remnant lipoproteins with atherogenic properties, how remnant lipoprotein clearance relates to atherosclerosis in people with diabetes remains unclear. In this issue of the $J C l$, Shimizu-Albergine et al. examined the effects of the basic leucine zipper transcription factor CREBH, which induces genes that activate LPL in mouse models of type I diabetes. Overexpression of a CREBH fragment reduced apolipoprotein C3 (APOC3) levels, which reduced plasma TGs. Notably, the TGs were lowered by a mechanism that was independent of LPL, and atherosclerosis was alleviated by enhanced lipoprotein remnant clearance as opposed to increased lipolysis of TG-rich lipoprotein precursors. A proinflammatory mechanism likely underlies the atherogenicity of remnant lipoproteins. These findings suggest that modifying CREBH expression in the liver may ameliorate atherosclerosis and, perhaps, other diabetes complications.
\end{abstract}

\section{Triglycerides and atherosclerosis}

Hypertriglyceridemia is the most common of dyslipidemias and often accompanies obesity, insulin resistance, type 2 diabetes, and poorly controlled type 1 diabetes. Hypertriglyceridemia correlates with decreases in HDL cholesterol and with increased risk of atherosclerosis. For many years, it was unclear whether elevated triglycerides (TGs) or low HDL was responsible for atherosclerosis. Through Mendelian randomization studies, it became possible to answer this question by analyzing the effect of variants that independently affect serum TGs versus HDL. TGs have emerged as the more likely causal agent (1, 2). However, this determination does not necessarily mean that TGs are the direct causal agent for atherosclerosis.

TGs are transported in the bloodstream primarily on chylomicrons, which are secreted by the intestine after a meal, or by very-low-density lipoproteins (VLDLs), which are continuously produced by the liver. In both cases, the TG cargo is hydrolyzed by lipoprotein lipase (LPL), which resides at the luminal surface of the capillary endothelium, giving rise to TGdepleted remnant lipoproteins. Chylomicron remnants are largely cleared by the liver, whereas VLDL remnants have two competing fates; they can be directly cleared by the liver like chylomicron remnants or further processed in the bloodstream to become LDL. Mutations that affect remnant clearance cause dysbetalipoproteinemia and atherosclerosis in humans (3). Remnant particles may be more atherogenic than LDL $(4,5)$. Remnant clearance largely depends on the binding of remnant-bound apolipoprotein $\mathrm{E}$ (APOE) to the LDL receptor, LDL receptor-related protein 1 (Lrp1), and syndecan-1

Related Article: https://doi.org/10.1172/JCl153285

Conflict of interest: The author has declared that no conflict of interest exists.

Copyright: @ 2021, American Society for Clinical Investigation.

Reference information: / Clin Invest. 2021;131(22):e154677. https://doi.org/10.1172/JCl154677.

(6). In mice, genetic deletion of Apoe leads to massive remnant accumulation and severe atherosclerosis (7) and indeed, the $A_{p o e^{-/}}$mouse is the most widely used animal model of atherosclerosis.

APOC3 is carried on TG-rich lipoproteins. Its levels correlate with serum TG levels. Treatment of hypertriglyceridemic humans (8) or mice (9) with an antisense oligonucleotide (ASO) against APOC3 markedly lowers serum TGs. APOC3 inhibits the access of LPL to its lipoprotein-borne TG substrate and also blocks the interaction of lipoprotein remnants with their cellular receptors (10). Diabetes leads to an increase in APOC3 levels (9), accounting in part for the hypertriglyceridemia frequently seen in people with diabetes. Mutations causing a lower level of APOC3 are associated with reduced risk of atherosclerosis. For example, a large study involving 110,970 individuals showed that those carrying mutations in $A P O C 3$ experienced an average TG reduction of $39 \%$ and had a $40 \%$ lower risk of atherosclerosis (11). Similarly, treatment of diabetic mice with an ASO against APOC3 lowers TGs and reduces atherosclerosis (9).

\section{CREBH expression affects lipoprotein metabolism and atherosclerosis}

In this issue of the JCI, Shimizu-Albergine et al. reported on the effects of the transcription factor CREBH on lipoprotein metabolism and atherosclerosis (12). SREBP and CREBH are synthesized as transmembrane proteins. CREBH is transported through the secretory pathway to the Golgi where it is cleaved by site-1 protease, the same enzyme that releases the active fragment of SREBP. To bypass the dependence on site- 1 protease, the authors expressed the active $\mathrm{N}$-terminal fragment of CREBH in vivo using adeno-associated virus in $L d l r$-KO mice with type 1 diabetes elicited by lymphocytic choriomeningitis virus glycoprotein-induced autoimmunity. 
A

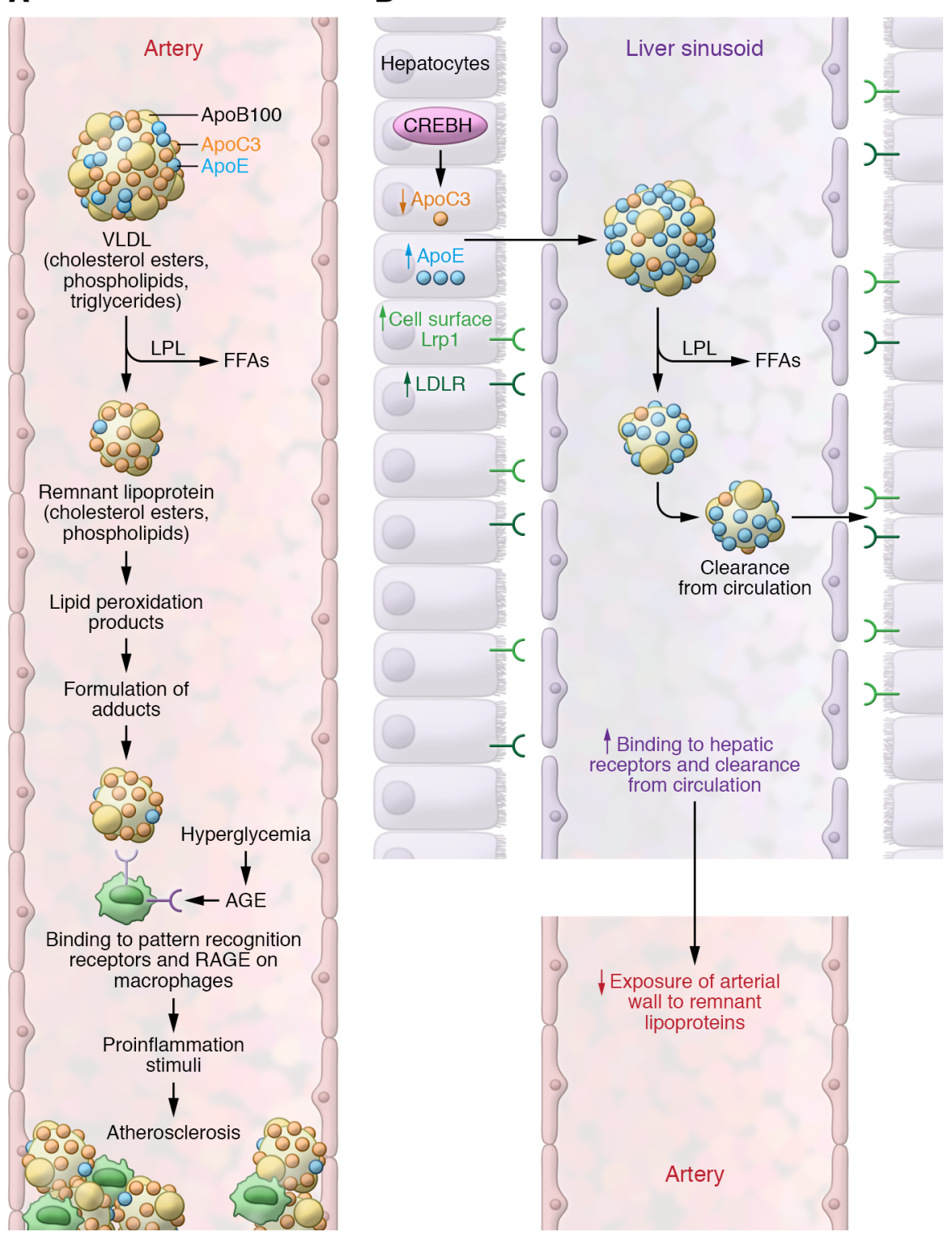

Figure 1. Enhanced lipoprotein remnant clearance ameliorates atherosclerosis in mice ectopically expressing CREBH. (A) In diabetes-related atherosclerosis, triglyceride-rich lipoproteins, VLDL, and chylomicrons (not shown) form remnant lipoproteins after lipoprotein lipase-mediated (LPL-mediated) triglyceride hydrolysis. Lipids associated with the lipoprotein particles are subject to oxidation. Oxidized lipids form complexes with proteins and bind to pattern recognition receptors on macrophages, inducing proinflammatory stimuli. Glucose can also form advanced glycosylation end products (AGEs), which bind to a distinct receptor for AGEs, termed RAGE. (B) Liver-specific expression of CREBH increases APOE, a ligand that mediates clearance of remnant particles and decreases APOC3, which can interfere with remnant clearance. CREBH also enhances the cell surface expression of the Lrp1 protein, one of several receptors that mediate remnant clearance. Clearance of remnant particles from the circulation reduces diabetes-related atherosclerosis. FFAs, free fatty acids.

APOC3 levels were markedly reduced by ectopic expression of the CREBH fragment, bringing about a substantial reduction in plasma TGs and to a lesser extent, plasma cholesterol. Contrary to published work, CREBH did not affect LPL levels in muscle or white adipose tissue, suggesting an LPL-independent mechanism for
TG lowering. Using Lrp1-knockdown and Apoe-KO mice, and examining plasma cholesterol versus TG, the authors surmised that the effect of CREBH on plasma TG was from APOE loading and remnant clearance via LDLR-family members, primarily Lrp1, rather than an activation of LPL. Shimizu-Albergine and colleagues concluded that type 1 diabetes-induced insulin deficiency increased APOC3 levels, decreased cell surface hepatic Lrp1 (insulin normally increases plasma membrane Lrp1), and caused hypertriglyceridemia. The studies are likely translatable to humans, as the authors went on to demonstrate that variants in the human homolog, CREB3L3, were associated with increased concentrations of remnant lipoproteins (12).

A major conclusion of ShimizuAlbergine et al. is that enhanced lipoprotein remnant clearance, rather than increased lipolysis of TG-rich lipoprotein precursors, ameliorated atherosclerosis in mice ectopically expressing CREBH (ref. 12 and Figure 1). This concept was supported by the much less severe atherosclerosis resulting from mutations that led to increased TG-rich lipoproteins rather than increased remnant particles (13). The results lend strong support to the concept, proposed nearly 50 years ago, that lipoprotein remnant particles are especially atherogenic (14).

\section{Conclusions and implications}

The precise mechanism underlying the atherogenicity of remnant lipoproteins is likely related to their proinflammatory effects. Oxidized lipids form oxidation-specific epitopes that stimulate the innate immune response (15). In addition, some of the fatty acids are substrates for proinflammatory prostanoids, such as PGE2 (16). Perhaps a detailed lipidomic analysis of the remnant lipoprotein particles in CREBHknock-down mice would help to identify additional proinflammatory lipids.

Glucose, like some oxidized lipids, is an aldehyde and also forms complexes with proteins, which bind to the receptor for advanced glycosylation end products (RAGE). RAGE plays a role in diabetes complications $(17,18)$ and inflammatory processes in pancreatic islets (19). Perhaps there is synergism between the glucoseinduced and oxidized lipid-induced adducts that leads to diabetes complications. Indeed, glucose- and lipid-modified epitopes can be detected in kidneys from patients with diabetic nephropathy (20, 21). This raises the exciting possibility that induction of CREBH may ameliorate diabetes complications like nephropathy.

Shimizu-Albergine et al. clearly demonstrate that effectively clearing lipoprotein remnants minimized their time in 
the circulation, their interaction with the vascular wall, and perhaps also their interaction with tissue macrophages and circulating monocytes (12). The specificity of CREBH in clearing lipoprotein remnants in the liver suggests that $\mathrm{CREBH}$ does not augment other processes (e.g., gluconeogenesis) that would discourage the development of drugs or gene-based therapies. These studies (12) provide a plausible path toward preventing or treating atherosclerosis and perhaps other diabetes complications through $\mathrm{CREBH}$ expression in the liver.

\section{Acknowledgments}

AA's research is supported by NIDDK grants DK101573 and DK125961.

Address correspondence to: Alan D. Attie, Department of Biochemistry, University of Wisconsin-Madison, 433 Babcock Dr., Madison, Wisconsin 53706, USA. Email: adattie@wisc.edu.

1. Voight BF, et al. Plasma HDL cholesterol and risk of myocardial infarction: a mendelian randomisation study. Lancet. 2012;380(9841):572-580.

2. Gill PK, et al. Genetics of hypertriglyceridemia and atherosclerosis. Curr Opin Cardiol. 2021;36(3):264-271.

3. Marais D. Dysbetalipoproteinemia: an extreme disorder of remnant metabolism. Curr Opin Lipidol. 2015;26(4):292-297.

4. Salinas CAA, Chapman MJ. Remnant lipoproteins: are they equal to or more atherogenic than LDL? Curr Opin Lipidol. 2020;31(3):132-139.

5 . Ginsberg HN, et al. Triglyceride-rich lipoproteins and their remnants: metabolic insights, role in atherosclerotic cardiovascular disease, and emerging therapeutic strategies-a consensus statement from the European Atherosclerosis Society [published online September 2, 2021]. Eur Heart J. https://doi.org/10.1093/ eurheartj/ehab551.

6. Foley EM, et al. Hepatic remnant lipoprotein clearance by heparan sulfate proteoglycans and low-density lipoprotein receptors depend on dietary conditions in mice. Arterioscler Thromb Vasc Biol. 2013;33(9):2065-2074.

7. Zhang SH, et al. Spontaneous hypercholesterolemia and arterial lesions in mice lacking apolipoprotein E. Science. 1992;258(5081):468-471.

8. Gaudet D, et al. Antisense inhibition of apolipoprotein C-III in patients with hypertriglyceridemia. N Engl J Med. 2015;373(5):438-447.

9. Kanter JE, et al. Increased apolipoprotein C3 drives cardiovascular risk in type 1 diabetes. JClin Invest. 2019;129(10):4165-4179.

10. Gordts PL, et al. ApoC-III inhibits clearance of triglyceride-rich lipoproteins through LDL family receptors. J Clin Invest. 2016;126(8):2855-2866.

11. Crosby J, et al. Loss-of-function mutations in APOC3, triglycerides, and coronary disease. N Engl J Med. 2014;371(1):22-31.

12. Shimizu-Albergine $\mathrm{M}$, et al. CREBH normalizes dyslipidemia and halts atherosclerosis in diabetes by decreasing circulating remnant lipoproteins. JClin Invest. 2021;131(22):e153285.
13. Basu D, Bornfeldt KE. Hypertriglyceridemia and atherosclerosis: using human research to guide mechanistic studies in animal models. Front Endocrinol (Lausanne). 2020;11:504.

14. Zilversmit DB. A proposal linking atherogenesis to the interaction of endothelial lipoprotein lipase with triglyceride-rich lipoproteins. Circ Res. 1973;33(6):633-638.

15. Binder CJ, et al. Innate sensing of oxidationpecific epitopes in health and disease. Nat Rev Immunol. 2016;16(8):485-497.

16. Kanter JE, et al. Diabetes promotes an inflammatory macrophage phenotype and atherosclerosis through acyl-CoA synthetase 1. Proc Natl Acad Sci U S A. 2012;109(12):E715-E724.

17. Egaña-Gorroño L, et al. Receptor for advanced glycation end products (RAGE) and mechanisms and therapeutic opportunities in diabetes and cardiovascular disease: insights from human subjects and animal models. Front Cardiovasc Med. 2020;7:37.

18. Nielsen TB, et al. Diabetes exacerbates infection via hyperinflammation by signaling through TLR4 and RAGE. mBio. 2017;8(4):e00818-17.

19. Abedini A, et al. RAGE binds preamyloid IAPP intermediates and mediates pancreatic $\beta$ cell proteotoxicity. JClin Invest. 2018;128(2):682-698.

20. Horie K, et al. Immunohistochemical colocalization of glycoxidation products and lipid peroxidation products in diabetic renal glomerular lesions. Implication for glycoxidative stress in the pathogenesis of diabetic nephropathy. JClin Invest. 1997;100(12):2995-3004.

21. Neale TJ, et al. Proteinuria in passive Heymann nephritis is associated with lipid peroxidation and formation of adducts on type IV collagen. J Clin Invest. 1994;94(4):1577-1584. 